\title{
Excluded-Volume Effects in Dilute Polymer Solutions. XIII. Effects of Chain Stiffness
}

\author{
Takashi NORISUYE and Hiroshi FuJITA \\ Department of Macromolecular Science, Osaka University, \\ Toyonaka, Osaka 560, Japan.
}

(Received August 7, 1981)

\begin{abstract}
The chain length dependence of mean-square radius of gyration $\left\langle S^{2}\right\rangle$ for several polymers, stiff or flexible, in dilute solution is compared with that for the Kratky-Porod (KP) wormlike chain, using representative literature data. In the region of $n_{\mathrm{K}}$, the number of Kuhn's statistical segments, below about $50,\left\langle S^{2}\right\rangle$ for all polymers examined is accurately described by Benoit-Doty's expression for the KP chain. Upward deviations from this expression occur at $n_{\mathrm{K}}$ of about 50, regardless of the chain stiffness. Thus, the critical chain length $L_{\mathrm{c}}$ for the onset of excluded-volume effect on the average dimensions of a polymer is approximately proportional to $q$, the persistence length of the polymer, with a proportionality factor of about 100 . The value of $L_{\mathrm{c}}$ estimated from this relation is at least one order of magnitude larger than that predicted theoretically by Yamakawa and Stockmayer.

KEY WORDS Stiff Chain / Wormlike Chain / Radius of Gyration / Excluded-Volume Effect / Persistence Length /
\end{abstract}

Previous work $^{1-4}$ on stiff or semiflexible polymers has shown that the mean-square radii of gyration $\left\langle S^{2}\right\rangle$ of this class of macromolecules in dilute solution can, in general, be described accurately by Benoit-Doty's theory ${ }^{5}$ for the KratkyPorod unperturbed wormlike chain ${ }^{6}$ (the KP chain). Ignoring the excluded-volume effect in dealing with stiff polymers is a reasonable approximation, since chain stiffness prevents segments from intramolecular collision. This approximation, however, should eventually break down as the chain length increases. In fact, our recent studies ${ }^{7-9}$ on stiff polymers, including poly(hexyl isocyanate) (PHIC), a typical example, demonstrate that the volume effect on $\left\langle S^{2}\right\rangle$ becomes observable when the chain length exceeds a certain value $L_{\mathrm{c}}$.

The purpose of the present paper is to investigate the correlation between chain stiffness and $L_{\mathrm{c}}$, using the $\left\langle S^{2}\right\rangle$ data obtained in these and other welldocumented studies. ${ }^{3,7-15}$ To make the data analysis quantitative, we express the stiffness of a given polymer in terms of the persistence length $q$ of the associated KP chain and treat deviations of measured $\left\langle S^{2}\right\rangle$ from the Benoit-Doty expression ${ }^{5}$ as due entirely to volume exclusion between chain segments.

\section{WORMLIKE CHAIN PARAMETERS}

Table I lists the reported values of $q$ and $M_{\mathrm{L}}$ (the molar mass per unit chain contour length) for the polymer + solvent systems chosen. The values for DNA have been reestimated by analyzing the $\left\langle S^{2}\right\rangle$ data of Godfrey and Eisenberg, ${ }^{3}$ using the method recently proposed by Murakami et al. ${ }^{8}$ and those for polystyrene in cyclohexane have been determined by the analysis described below.

The data of Ballard et al. ${ }^{13}$ are plotted as $\left\langle S^{2}\right\rangle /$ $M_{w}$ against $\log M_{w}$ in Figure 1 , where $M_{w}$ is the weight-average molecular weight of the polymer. This graph also includes the light scattering data of Miyaki et al. ${ }^{14}$ and Oyama et al. ${ }^{15}$ for higher molecular weight samples in the same solvent. The data points are fitted by a smooth curve, which rises sharply in the region of $M_{w}$ below $10^{4}$ and then levels off at a constant value. The initial rise is a reflection of a finite chain stiffness of the polystyrene molecule.

Benoit-Doty's expression for $\left\langle S^{2}\right\rangle$ of the KP chain may be written 


$$
\frac{\left\langle S^{2}\right\rangle}{(2 q)^{2}}=\frac{n_{\mathrm{K}}}{6}-\frac{1}{4}+\frac{1}{4 n_{\mathrm{K}}}-\frac{1}{8 n_{\mathrm{K}}^{2}}\left[1-\exp \left(-2 n_{\mathrm{K}}\right)\right]
$$

where

$$
n_{\mathrm{K}}=M /\left(2 q M_{\mathrm{L}}\right)
$$

with $M$ the molecular weight of the chain. The quantity $n_{\mathrm{K}}$ is the number of Kuhn's statistical segments in the chain and is related to the chain contour length $L$ by $L=2 q n_{\mathrm{K}}$. The $n_{\mathrm{K}}$ value corresponding to the critical chain length $L_{\mathrm{c}}$ is denoted by $n_{\mathrm{K}}{ }^{\mathrm{c}}$.

From eq 1 it follows that

$$
\begin{gathered}
C_{\infty}{ }^{\prime} \equiv \lim _{n_{\mathrm{K}} \rightarrow \infty}\left\langle S^{2}\right\rangle / M=q / 3 M_{\mathrm{L}} \\
M_{1 / 2}=3.54 q M_{\mathrm{L}}
\end{gathered}
$$

where $M_{1 / 2}$ denotes the value of $M$ at which $\left\langle S^{2}\right\rangle /$ $M$ equals $C_{\infty}{ }^{\prime} / 2$. From Figure 1 we find $C_{\infty}{ }^{\prime}=8.6$ $( \pm 0.2) \times 10^{-18} \mathrm{~cm}^{2}$ and $M_{1 / 2}=1400( \pm 200)$. These

\begin{tabular}{|c|c|c|c|c|}
\hline \multirow{2}{*}{ Polymer } & $q$ & $M_{\mathrm{L}}$ & \multirow{2}{*}{$n_{\mathrm{K}}^{\mathrm{c}}$} & \multirow{2}{*}{$v$} \\
\hline & $\mathrm{nm}$ & $\mathrm{nm}^{-1}$ & & \\
\hline Schizophyllan $^{a}$ & 150 & 2170 & - & 一 \\
\hline DNA $^{b}$ & 68 & 1970 & - & - \\
\hline $\mathrm{PHIC}^{\mathrm{c}}$ & 42 & 715 & 50 & 1.2 \\
\hline PTDPd & 6.4 & 350 & - & - \\
\hline \multirow[t]{2}{*}{ PPDP $^{\mathrm{e}}$} & 3.7 & 330 & \multirow{2}{*}{80} & \multirow{2}{*}{$1.0_{6}$} \\
\hline & $3.3^{\mathrm{f}}$ & $330^{\mathrm{f}}$ & & \\
\hline PIDPg & 3.1 & 330 & 50 & 1.2 \\
\hline $\mathrm{PC}^{\mathrm{h}}$ & 1.8 & 260 & 60 & 1.1 \\
\hline Polystyrene & $1.0^{\mathrm{i}}$ & $390^{i}$ & $30^{\mathrm{j}}$ & $1.2^{\mathrm{j}}$ \\
\hline
\end{tabular}

Table I. Parameters associated with the dimensions of unperturbed and perturbed wormlike chains

a In $0.01 \mathrm{~N} \mathrm{NaOH}$ at $25^{\circ} \mathrm{C}$ (ref 12).

b In $0.2 \mathrm{M} \mathrm{NaCl}+2 \mathrm{mM}$ EDTA $+2 \mathrm{mM} \mathrm{Na}-\mathrm{PO}_{4}(\mathrm{pH} 7)$ at $25^{\circ} \mathrm{C}$ (ref 3 ).

c Poly(hexyl isocyanate) in hexane at $25^{\circ} \mathrm{C}$ (ref 8).

d Poly(terephthaloyl-trans-2,5-dimethylpiperazine) in trifluoroethanol at $25^{\circ} \mathrm{C}$ (ref 11).

e Poly(phthaloyl-trans-2,5-dimethylpiperazine) in chloroform at $25^{\circ} \mathrm{C}$ (ref 10 ).

${ }^{f}$ In $N$-methyl-2-pyrrolidone at $25^{\circ} \mathrm{C}$ (ref 10$)$.

g Poly(isophthaloyl-trans-2,5-dimethylpiperazine) in trifluoroethanol at $25^{\circ} \mathrm{C}$ (ref 9).

h Bisphenol A polycarbonate in tetrahydrofuran at $25^{\circ} \mathrm{C}$ (ref 7).

${ }^{i}$ In cyclohexane at the theta temperature (ref 13-15).

j In benzene at $25^{\circ} \mathrm{C}$ (ref 14$)$.

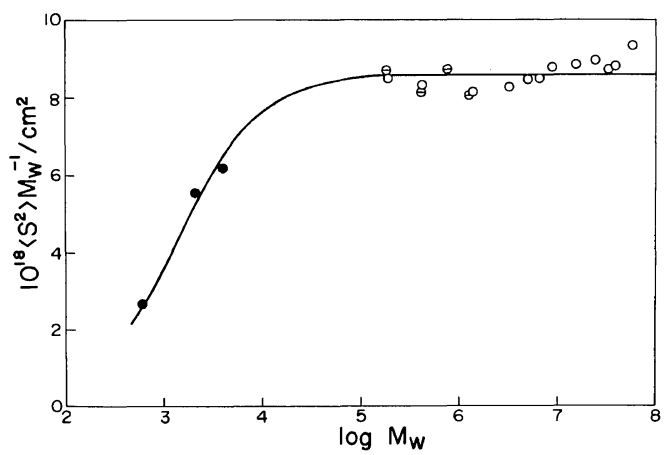

Figure 1. Plot of $\left\langle S^{2}\right\rangle / M_{w} v s . \log M_{w}$ for polystyrene in cyclohexane at the theta temperature. (O), Ballard et al. ${ }^{13} ;(\bigcirc)$, Miyaki et al. ${ }^{14} ;(\ominus)$, Oyama et al. ${ }^{15}$ The curve represents eq 1 with $q=1.0 \mathrm{~nm}$ and $M_{\mathrm{L}}=390 \mathrm{~nm}^{-1}$.

values, substituted into eq 3 and 4 , give $q=1.0$ $( \pm 0.1) \mathrm{nm}$ and $M_{\mathrm{L}}=390( \pm 30) \mathrm{nm}^{-1}$, which are presented in Table I.

The solid curve in Figure 1 has been calculated from eq 1 with $q=1.0 \mathrm{~nm}$ and $M_{\mathrm{L}}=390 \mathrm{~nm}^{-1}$. Its good fit to the data points substantiates that the unperturbed wormlike chain is a reasonable model for polystyrene in cyclohexane at the theta temperature. We note that the $M_{\mathrm{L}}$ value of $390 \mathrm{~nm}^{-1}$ is close to $408 \mathrm{~nm}^{-1}$, computed on the assumption that the polystyrene molecule assumes all-trans conformation. ${ }^{16}$

\section{CRITICAL CHAIN LENGTH}

If eq 1 holds, a reduced mean-square radius of gyration $\left\langle S^{2}\right\rangle /(2 q)^{2}$ should be a universal function of $n_{\mathrm{K}}$. To check this prediction, we made log-log plots of $\left\langle S^{2}\right\rangle /(2 q)^{2}$ against $n_{\mathrm{K}}$ using the reported data for the systems listed in Table I. The results are shown in Figure 2. The points for the system polystyrene + benzene in this figure have been included, assuming that the $q$ and $M_{\mathrm{L}}$ for polystyrene in cyclohexane are also applicable to a good solvent benzene.

All plotted points for $n_{\mathrm{K}}$ below about 50 are seen to fall on the solid curve actually representing eq 1 . This implies that regardless of chain stiffness and solvent, polymers with $n_{\mathrm{K}}$ less than 50 are not perturbed by excluded-volume effect and their statistical radii obey eq 1 . The data points start deviating upward from the solid curve in a region of $n_{\mathrm{K}}$ around 50 and follow the dashed lines as indicated, except for those of polystyrene in cyclohexane. It is 


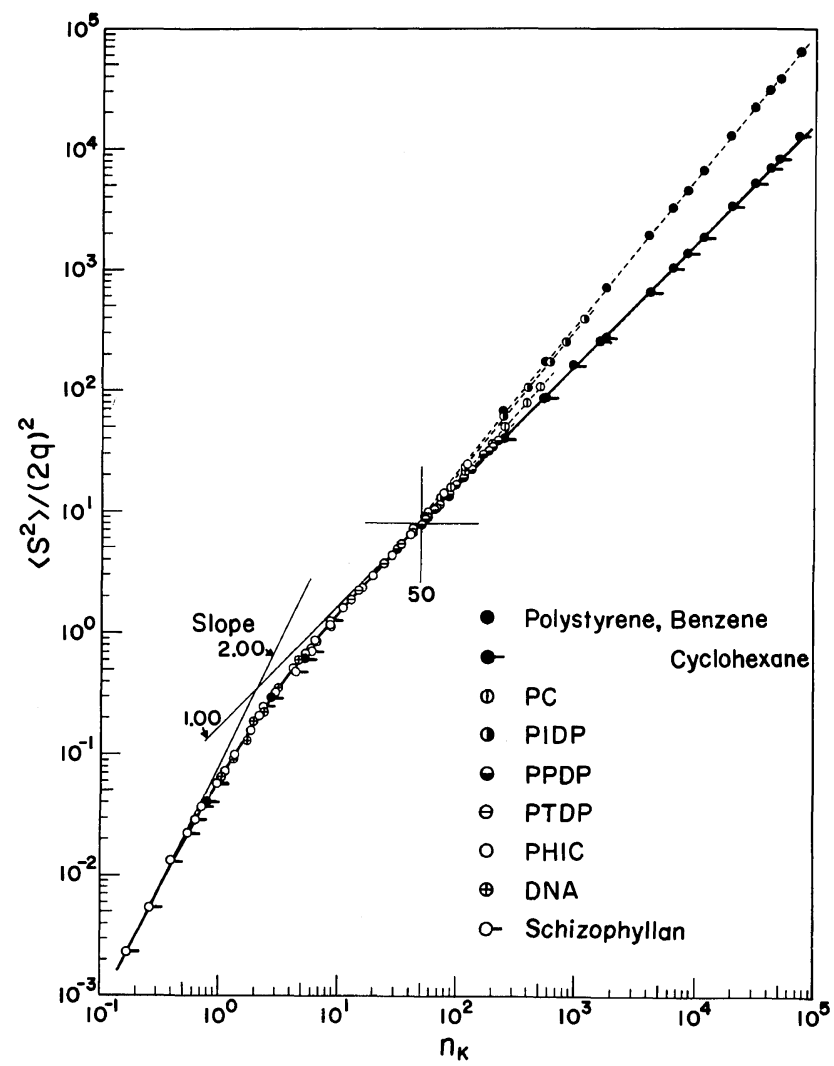

Figure 2. Reduced mean-square radii of gyration plotted against the number of Kuhn's statistical segments for different polymer + solvent systems. The solid line represents eq 1 .

reasonable to assume these deviations to be due primarily to volume effects and to define $n_{\mathrm{K}}$ at the intersection of the dashed and solid lines as the critical segment number $n_{\mathrm{K}}{ }^{\mathrm{c}}$ for the onset of volume effect in a given polymer.

In Figure 3, the data in the region of $n_{\mathrm{K}}$ between 30 and 1100 are displayed on a larger scale, along with those for polystyrene in cyclohexane omitted. The values of $n_{\mathrm{K}}{ }^{\mathrm{c}}$ estimated from this figure are given in the fourth column of Table I. They are approximately constant, ranging from 30 to 80 and showing no discernible correlation with chain stiffness. In fact, the $q$ values of polystyrene and PHIC differ as much as about 40 times. The effect of solvent on $n_{\mathrm{K}}{ }^{\mathrm{c}}$ is not clear by these limited data. Summarizing, we may write the following empirical relation between the critical chain length $L_{\mathrm{c}}=2 q n_{\mathrm{K}}{ }^{\mathrm{c}}$ and the persistence length $q$ :

$$
L_{\mathrm{c}} \sim 100 q
$$

It should be noted that the proportionality factor in eq 5 is at least one order of magnitude higher than that estimated from the theory of Yamakawa and Stockmayer ${ }^{17}$ who developed a first-order perturbation theory for the volume effect in the KP chain. In other words, chain stiffness prevents intramolecular contact of chain segments more strongly than is predicted by this theory.

The dashed lines in Figure 3, though giving approximately the same values of $n_{\mathrm{K}}{ }^{\mathrm{c}}$, have distinctly different slopes $v$ (see the fifth column of Table I) for different polymer + solvent systems. Apparently, no correlation can be seen between $v$ and $q$. If inferred from our knowledge on flexible polymers, the major factor for $v$ would be the binary cluster integral $\beta$ for segment-segment interactions.

Poly(methyl methacrylate) (PMMA) is known as a flexible polymer, but the data ${ }^{18-24}$ shown in Figure 4 indicate that it is stiff at very low molecular weight, just as is another flexible polymer poly- 
styrene of comparative molecular weights. In Figure 4 , the slope of the solid curve fitting the acetone data first decreases to 0.5 , stays at this value over a certain range (from $10^{3}$ to $2 \times 10^{4}$, approximately), and then changes rather discontinuously to a higher

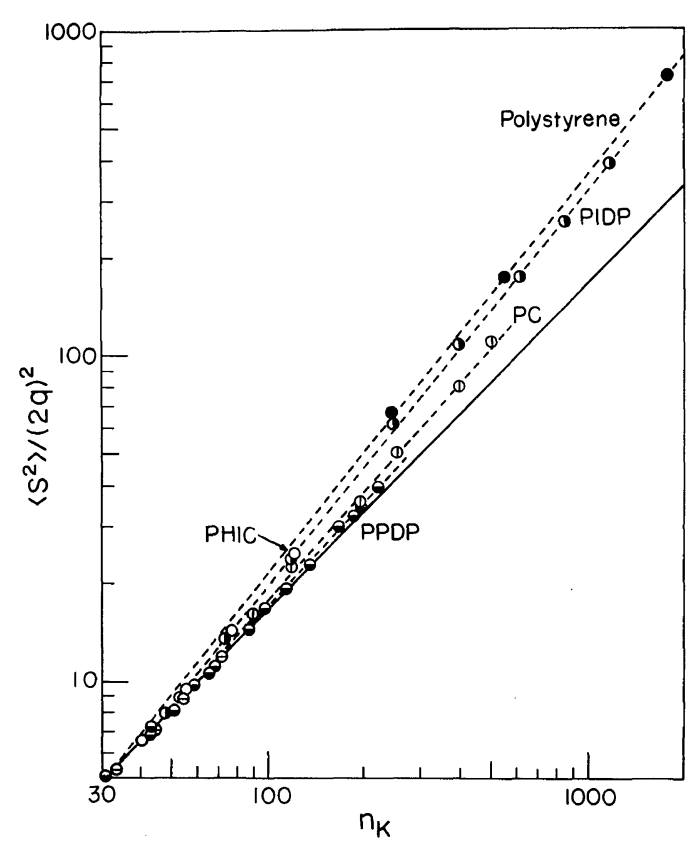

Figure 3. Enlarged graph of Figure 3. The data for polystyrene in cyclohexane are not displayed. value 0.6 . Interestingly, the portion with the slope of 0.5 merges with the extension of the dashed line fitting the data points ${ }^{25,26}$ for $n$-butyl chloride at $35^{\circ} \mathrm{C}$, a theta solvent for PMMA. Thus, we may consider that PMMA in acetone, a good solvent for this polymer, undergoes no excluded-volume effect until the molecular weight reaches $2 \times 10^{4}$ and behaves like a stiff chain at molecular weights lower than $10^{3}$.

The chemical structure of PMMA suggests that its $M_{\mathrm{L}}$ be in the range between 330 and $400 \mathrm{~nm}^{-1}$. Yamakawa and Fujiii ${ }^{27}$ estimated $M_{\mathbf{L}}$ for atactic and syndiotactic PMMA to be $370 \mathrm{~nm}^{-1}$, by fitting the expression for the mean-square end-to-end distance $\left\langle R^{2}\right\rangle$ of a helical wormlike chain to the values of $\left\langle R^{2}\right\rangle$ computed by Yoon and Flory ${ }^{28}$ on the rotational isomeric state models. If YamakawaFujii's value of $M_{\mathrm{L}}$ and the relation $\left\langle S^{2}\right\rangle^{1 / 2}=$ $2.2 \times 10^{-9} M_{w}{ }^{1 / 2}(\mathrm{~cm})$ representing the dashed line in Figure 4 are substituted into eq 1 with eq 2 and only the leading term in eq 1 is retained, a value of 0.53 $\mathrm{nm}$ is obtained for $q$. With these values for $q$ and $M_{\mathrm{L}}$, we find 50 for $n_{\mathrm{K}}$ at the above-mentioned critical molecular weight $2 \times 10^{4}$. This $n_{\mathrm{K}}{ }^{\mathrm{c}}$ value is very consistent with those estimated above for other polymers.

The dot-dash line in Figure 4 represents eq 1 with $q=0.53 \mathrm{~nm}$ and $M_{\mathrm{L}}=370 \mathrm{~nm}^{-1}$. It does not fit the acetone data for $M_{w}$ below $10^{4}$. This fact is in agreement with the finding of Kirste and

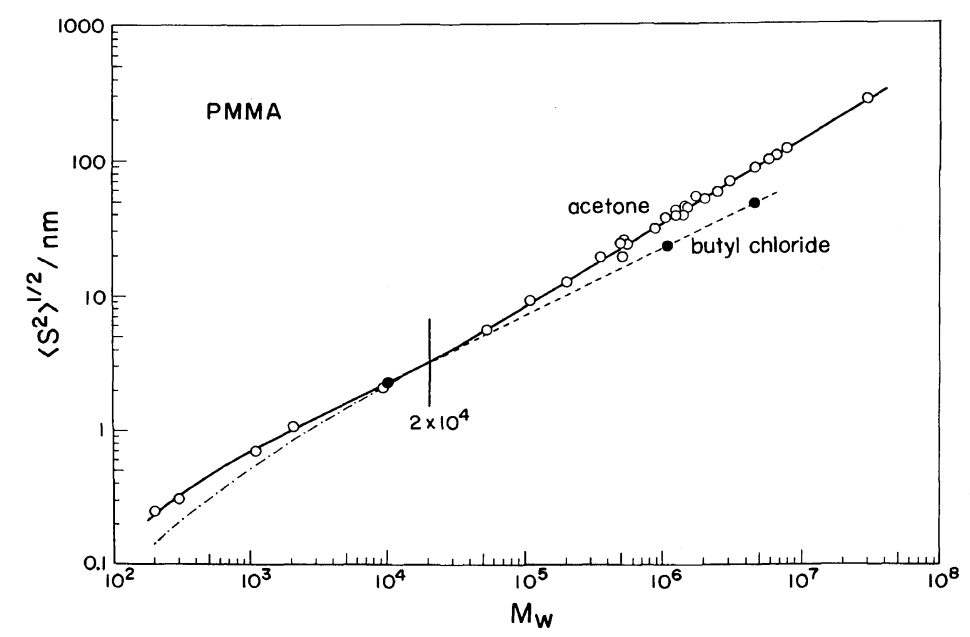

Figure 4. Molecular weight dependence of $\left\langle S^{2}\right\rangle^{1 / 2}$ for poly(methyl methacrylate) in acetone, a good solvent, and in $n$-butyl chloride $\left(35^{\circ} \mathrm{C}\right)$, a theta solvent. ${ }^{18-26}$ 
Wunderlich ${ }^{23}$ that low molecular weight PMMA in acetone cannot exactly be modeled by the KP chain, and illustrates the limitation of the KP chain as a model for actual polymer molecules.

\section{REFERENCES}

1. R. G. Kirste, Discuss. Faraday Soc., 49, 51 (1970).

2. M. N. Berger, J. Macromol. Sci., Rev. Macromol. Chem., C9, 269 (1973).

3. J. E. Godfrey and H. Eisenberg, Biophys. Chem., 5, 301 (1976).

4. M. Arpin and C. Strazielle, Polymer, 18, 591 (1977).

5. H. Benoit and P. Doty, J. Phys. Chem., 57, 958 (1953).

6. O. Kratky and G. Porod, Rec. Trav. Chim., 68, 1106 (1949).

7. T. Tsuji, T. Norisuye, and H. Fujita, Polym. J., 7, 558 (1975).

8. H. Murakami, T. Norisuye, and H. Fujita, Macromolecules, 13, 345 (1980).

9. J. Sadanobu, T. Norisuye, and H. Fujita, Polym. J., 13, 75 (1981).

10. M. Motowoka, T. Norisuye, and H. Fujita, Polym. J., 9, 613 (1977).

11. M. Motowoka, H. Fujia, and T. Norisuye, Polym. J., 10, 331 (1978).

12. Y. Kashiwagi, T. Norisuye, and H. Fujita, Macromolecules, 14, 1220 (1981).
13. B. G. H. Ballard, M. G. Rayner, and J. Schelten, Polymer, 17, 349 (1976).

14. Y. Miyaki, Y. Einaga, and H. Fujita, Macromolecules, 11, 1180 (1979); Y. Miyaki, Ph.D. Thesis, Osaka University, 1981.

15. T. Oyama, K. Shiokawa, and K. Baba, Polym. J., 13, 167 (1981).

16. H. Yamakawa, Macromolecules, 10, 692 (1977).

17. H. Yamakawa and W. H. Stockmayer, J. Chem. Phys., 57, 2843 (1972).

18. H. J. Cantow and G. V. Schulz, Z. Phys. Chem. (Frankfurt), 2, 117 (1954).

19. H. J. Cantow and O. Bodmann, Z. Phys. Chem. (Frankfurt), 3, 65 (1955).

20. G. V. Schulz and H. Craubner, Z. Elektrochem., 63, 301 (1959).

21. E. Cohn-Ginsberg, T. G Fox, and H. F. Mason, Polymer, 3, 97 (1962).

22. W. Wunderlich, Makromol. Chem., 108, 315 (1967).

23. R. G. Kirste and W. Wunderlich, Z. Phys. Chem. (Frankfurt), 58, 133 (1968).

24. Y. Kashiwagi, Y. Einaga, and H. Fujita, Polym. J., 12, 271 (1980).

25. G. V. Schulz and R. G. Kirste, Z. Phys. Chem. (Frankfurt), 30, 171 (1961).

26. R. G. Kirste, W. A. Kruse, and K. Ibel, Polymer, 16, 120 (1975).

27. H. Yamakawa and M. Fujii, J. Chem. Phys., 64, 5222 (1976).

28. D. Y. Yoon and P. J. Flory, Polymer, 16, 645 (1975). 